\title{
Efficient Complex Sphere Decoding for MC-CDMA Systems
}

\author{
Ricardo Santiago Mozos and M. Julia Fernández-Getino García
}

\begin{abstract}
Maximum Likelihood (ML) joint detection of MultiCarrier Code Division Multiple Access (MC-CDMA) systems can be effi iently implemented with a Sphere Decoding (SD) algorithm. In this paper, we examine the application of complex instead of real SD to detect MC-CDMA, which solves many problems in a more elegant manner and extends SD adaptability to any constellation. We f rst propose a new complex SD algorithm whose effi iency is based on not requiring an estimate of the initial search radius but selecting the Babai Point as the initial sphere radius instead; also, effi ient strategies regarding sorting the list of possible lattice points are applied. Indeed, complex SD allows complex matrix operations which are faster than real counterparts in double dimension. Next, a novel lattice representation for the MC-CDMA system is introduced, which allows optimum multiuser detection directly from the received signal. This avoids noise whitening operation, and also despreading and equalization procedures are not required further at the receiver side.
\end{abstract}

Index Terms-Multi carrier code division multiple access, maximum likelihood decoding, sphere decoding, multiuser detection.

\section{INTRODUCTION}

$\mathbf{T}$ HE combination of the principles of Code Division Multiple Access (CDMA) with Multi-Carrier modulation, called MC-CDMA and f rst proposed in 1993 [1], [2], is based on applying Orthogonal Frequency Division Multiplexing (OFDM) technique to a multiuser direct sequence DS-CDMA signal. In this paper, the synchronous downlink is addressed, which applies to the case of a single broadcaster simultaneously sending data symbols over an MC-CDMA link, as well as to the case of symbols being transmitted to multiple users which are multiplexed onto a common multicarrier signal. In any case, the technique consists of spreading each frequencydomain complex symbol over all subcarriers, and therefore transmitting a portion of every symbol on each subcarrier; if certain subcarriers are lost due to high frequency selectivity, all symbols can still be retrieved. The term multiuser interference (MUI) will be employed for any mutual interference between different complex symbols due to frequency dispersion of the channel, even though in the broadcast scenario all frequencydomain complex symbols belong to the same user. To combat this multiuser interference due to the loss of orthogonality, various multiuser detection techniques have been proposed [3],

This work has been partly funded by the Spanish government with national project MACAWI (TEC 2005-07477-c02-02) and project MAMBO (UC3M-TEC-05027).

The authors are with the Department of Signal Theory and Communications, Universidad Carlos III de Madrid, Avda. de la Universidad 30, 28911 Leganés-Madrid, Spain (email: \{rsmozos, mjulia\}@tsc.uc3m.es).
[4], [5]; optimum multiuser detection, based on joint detection using a Maximum Likelihood (ML) criterion, has a prohibitive complexity that grows exponentially with the number of users and the number of bits per modulation symbol [1].

The Sphere Decoding (SD) algorithm is an optimum ML algorithm [6] but with lower expected complexity [7], [8]. It was introduced in [9], frst used in communications problems in [10] and later applied to lattice code decoding in fading channels in [11]. These previous works deal with real SD algorithms; a complex sphere decoder algorithm was frst proposed by Hochwald [12]. It is worth mentioning a good introduction to SD algorithm by Agrell [6].

Brunel introduced optimum and sub-optimum ML multiuser detection schemes based on SD for MC-CDMA [13]. However, this scheme shows some complexity issues: its application is limited to rectangular Quadrature Amplitude Modulation (QAM) constellations ${ }^{1}$, the received signal has to be divided into in-phase and quadrature components to handle real symbols and so the dimension of the lattice is then twice the dimension of the received symbol, and finall, this SD method needs an estimate of the initial search radius for the sphere. In addition, Brunel's lattice model requires noise whitening before the decoding procedure.

To reduce the complexity, we frst propose a new complex $\mathrm{SD}$ algorithm that performs an eff cient search on the lattice points and then apply it to MC-CDMA systems. Indeed, a complex algorithm allows complex matrix operations which are faster than real counterparts in double dimension [14], [15]. Moreover, a new lattice model for the MC-CDMA system is introduced, which avoids any whitening process. We evaluate the proposed detection scheme in a high frequencyselective channel and compare SD with some sub-optimal methods such as Equal Gain Combining (EGC), Minimum Mean-Squared Error (MMSE), and Minimum Mean-Squared Error per User (MMSE-U) [16], [17], [18] in full and non-

Section II the ML-LDIVIA transmission system is described. Complex sphere decoding is investigated in Section III. Optimum detection of an MC-CDMA system with a sphere decoding algorithm and its corresponding lattice description are addressed in Section IV; also a new lattice model is proposed in this Section. In Section V simulation results are presented and, fi ally, some conclusions are drawn in Section VI.

\section{MODEL DESCRIPTION OF MC-CDMA}

Let us consider a synchronous MC-CDMA system with $K$ users and $L$ subcarriers, $K \leq L$. Every $k$-th user, $1 \leq k \leq K$,

\footnotetext{
${ }^{1}$ Checking invalid points is needed when dealing with constellations other than rectangular QAM, that is, constellations which do not comprise all combinations of real and imaginary alphabets.
} 


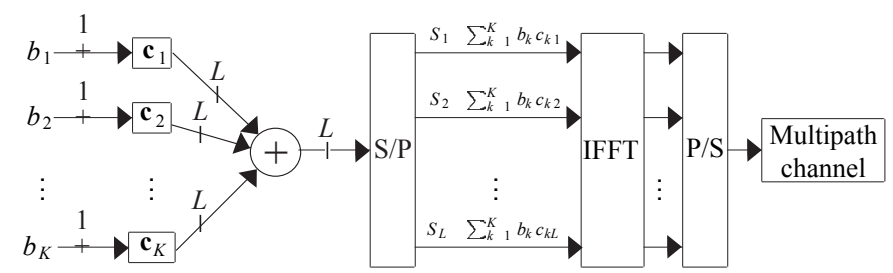

Fig. 1. MC-CDMA transmission model for downlink.

transmits a complex symbol $b_{k}$ per time slot; this symbol is spread using a complex spread signature $\mathbf{c}_{k}=\left\{c_{k 1}, \ldots, c_{k L}\right\}$ of length $L$ taken from an orthonormal set $\left.\left(<\mathbf{c}_{k}, \mathbf{c}_{j}\right\rangle=\delta_{k j}\right)$. Each chip of the resulting vector after spreading $\left(\mathbf{c}_{k} b_{k}\right)$ is then transmitted onto a different subcarrier. Thus, at $\ell$-th subcarrier, $1 \leq \ell \leq L$, the sum of chips from all users $S_{\ell}=\sum_{k=1}^{K} b_{k} c_{k \ell}$ is sent. A block diagram for the MC-CDMA transmission system is sketched in Figure 1. Assuming that every subcarrier experiences almost $\mathrm{f}$ at-fading, the channel is denoted as $L \times L$ diagonal matrix $\mathbf{H}$ where $H_{\ell \ell}$ is the single complex channel coefficien per $\ell$-th subcarrier.

The received signal in frequency-domain is the $L \times 1$ vector $\mathbf{r}$ given by

$$
\mathbf{r}=\mathbf{H C b}+\mathbf{n}
$$

where $\mathbf{C}$ is the $L \times K$ spreading matrix with $(\mathbf{C})_{k}=\mathbf{c}_{k}$, where $(\cdot)_{k}$ denotes the $k$-th column of a matrix; $K \times 1$ vector b contains the user symbols and $L \times 1$ vector $\mathbf{n}$ represents the Additive White Gaussian Noise (AWGN). At the receiver, the signal is usually equalized and despread to obtain the $K \times 1$ output vector $\mathbf{y}$, which aims to be a good estimate of $\mathbf{b}$,

$$
\mathbf{y}=\mathbf{C}^{*} \mathbf{G r}=\mathbf{C}^{*} \mathbf{G H C b}+\mathbf{C}^{*} \mathbf{G n}
$$

where $L \times L$ equalization matrix $\mathbf{G}$ compensates the channel to restore orthogonality of the spreading codes, and the signal is later despread using $\mathbf{C}^{*} ;(\cdot)^{*}$ denotes conjugate transpose matrix operation. Note that after these processes, the noise term $\left(\mathbf{C}^{*} \mathbf{G n}\right)$ is not AWG anymore; this issue is analyzed later in Section IV.

\section{COMplex Sphere DeCODING}

Sphere Decoding (SD) is an optimum technique to jointly detect the symbols for all users using ML criterion. The principal advantage of SD over traditional joint detection is that the search is only performed inside a sphere with a certain radius and thus the expected complexity is roughly cubic with the number of users at moderate Signal-to-Noise Ratio (SNR) values. The analysis provided herein is, in large part, an extension of Hochwald's complex sphere decoder [12]. Let a generic transmission system be described as

$$
\mathbf{r}=\mathbf{H} \mathbf{s}+\mathbf{n}
$$

where $L \times 1$ vector $\mathbf{r}$ represents the system output, $\mathbf{H}$ is the $L \times K$ channel matrix, $K \times 1$ vector $\mathbf{s}$ is the transmitted signal sequence and $L \times 1$ vector $\mathbf{n}$ represents AWGN. Therefore, $\mathbf{s}$ can be estimated from $\mathbf{r}$ using SD algorithm. In joint ML decoding, we look for $\hat{\mathbf{s}}_{\mathrm{ML}}$ such as

$$
\hat{\mathbf{s}}_{\mathrm{ML}}=\arg \min _{\mathbf{s} \in \Lambda}\|\mathbf{r}-\mathbf{H} \mathbf{s}\|^{2}=\arg \min _{\mathbf{s} \in \Lambda}(\hat{\mathbf{s}}-\mathbf{s})^{*} \mathbf{H}^{*} \mathbf{H}(\hat{\mathbf{s}}-\mathbf{s})
$$

where $\hat{\mathbf{s}}=\left(\mathbf{H}^{*} \mathbf{H}\right)^{-1} \mathbf{H}^{*} \mathbf{r}$ is the transformed received point and the center of the search sphere and $\Lambda$ is the lattice generated by $\mathbf{H}$. Indeed, we can think of the columns of $\mathbf{H}$ as basis vectors of a lattice lying in a $L$ dimensional space; vector $\mathbf{s}$ can be seen as the coordinates of a lattice point.

To solve this problem eff ciently Cholesky factorization is employed to fnd an upper triangular $\mathbf{U}$ with $u_{i i}$ real and positive such $\mathbf{U}^{*} \mathbf{U}=\mathbf{H}^{*} \mathbf{H}$ and the search is only performed for the $\mathbf{s}$ that lie inside a sphere. So (3) can be written as

$$
\begin{aligned}
\hat{\mathbf{s}}_{\mathrm{ML}} & =\arg \min _{\mathbf{s} \in \Lambda}(\hat{\mathbf{s}}-\mathbf{s})^{*} \mathbf{U}^{*} \mathbf{U}(\hat{\mathbf{s}}-\mathbf{s}) \\
& =\arg \min _{\mathbf{s} \in \Lambda} \sum_{i=1}^{K} u_{i i}^{2}\left|\hat{s}_{i}-s_{i}+\sum_{j=i+1}^{K} \frac{u_{i j}}{u_{i i}}\left(\hat{s}_{j}-s_{j}\right)\right|^{2} \leq r^{2}
\end{aligned}
$$

where $r$ denotes the radius of the sphere where the search is performed $^{2}$. The search starts setting $i=K$ in (4), which provides inequality $\left|\hat{s}_{K}-s_{K}\right| \leq \frac{r}{u_{K K}}$. From the symbol list that satisfie the former inequality, a valid symbol $s_{K}$ is selected for $K$ dimension. Once $s_{K}$ is fi ed, a valid symbol $s_{K-1}$ can be selected for $K-1$ dimension from the symbol list that satisfie the inequality obtained by setting $i=K-1$ in (4). The search continues in the same way for the rest of dimensions. If there are not valid symbols that fulf 11 (4) in a certain dimension for all combinations of symbols in previous dimensions, then the search radius must be increased and the search starts again.

In the following, we will develop recursive equations for a quick evaluation of the list of possible constellation symbols in each dimension that satisfy (4); at the end, we aim to obtain a set of equations for an eff cient search algorithm.

Without loss of generality, a Phase Shift Keying (PSK) constellation is assumed; a more complex constellation can be represented considering different circumferences with the appropriate radii. The intersection between the circumference of the PSK constellation and the search disk is an arc whose sweep can be found analytically. Let $s_{K}=r_{c} e^{j \theta_{K}}$, where $\theta_{K} \in\left\{0, \frac{2 \pi}{2^{M_{c}}}, \ldots, \frac{2 \pi\left(2^{M_{c}}-1\right)}{2^{M_{c}}}\right\}$ are the angles of a $2^{M_{c_{-}}}$ PSK constellation. To simplify the notation let $\xi_{i}=\hat{s}_{i}-s_{i}$, $q_{i i}=u_{i i}^{2}, q_{i j}=u_{i j} / u_{i i}$ and

$$
R_{i}=\frac{1}{q_{i i}}\left(r^{2}-\sum_{l=i+1}^{K} q_{l l}\left|\xi_{l}+\sum_{j=l+1}^{K} q_{l j} \xi_{j}\right|^{2}\right)
$$

which can be efficientl computed using

$$
\begin{aligned}
T_{i} & =r^{2}-\sum_{l=i+1}^{K} q_{l l}\left|\xi_{l}+\sum_{j=l+1}^{K} q_{l j} \xi_{j}\right|^{2} \quad S_{i}=\sum_{j=i+1}^{K} q_{i j} \xi_{j} \\
T_{i-1}=T_{i}-q_{i i}\left|\xi_{i}+S_{i}\right|^{2} & R_{i}=\frac{T_{i}}{q_{i i}}
\end{aligned}
$$

By defi ing $s_{i}^{\prime}=\hat{s}_{i}+S_{i}=r_{c, i}^{\prime} e^{j \theta_{i}^{\prime}}$, we can write for the $i$ th

\footnotetext{
${ }^{2}$ In [12] there is a typo in Equation (23) which is Equation (4) here.
} 
sum term in (4)

$$
\begin{gathered}
\left|s_{i}^{\prime}-s_{i}\right|^{2}=r_{c}^{2}+r_{c, i}^{\prime 2}-2 r_{c} r_{c, i}^{\prime} \cos \left(\theta_{i}-\theta_{i}^{\prime}\right) \leq R_{i} \\
\cos \left(\theta_{i}-\theta_{i}^{\prime}\right) \geq \frac{1}{2 r_{c} r_{c, i}^{\prime}}\left[r_{c}^{2}+r_{c, i}^{\prime 2}-R_{i}\right]=\eta_{i}
\end{gathered}
$$

Therefore, if $-1 \leq \eta_{i} \leq 1$ the range of allowable points for the $i$-th dimension is

$$
\left\lceil\frac{2^{M_{c}}}{2 \pi}\left(\hat{\theta}_{i}-\cos ^{-1} \eta_{i}\right)\right] \leq \frac{2^{M_{c}}}{2 \pi} \theta_{i} \leq\left\lfloor\frac{2^{M_{c}}}{2 \pi}\left(\hat{\theta}_{i}+\cos ^{-1} \eta_{i}\right)\right\rfloor
$$

where $\lceil\cdot\rceil$ and $\lfloor\cdot\rfloor$ operators return the smallest integer greater than or equal to, and, the largest integer less than or equal to their respective arguments. When a point inside the sphere is obtained, the distance from the received point can be determined using

$$
d^{2}=T_{K}-T_{0}=T_{K}-T_{1}+q_{11}\left|\xi_{1}+S_{1}\right|^{2} .
$$

Next, the search radius is updated as $r^{2}=d^{2}$ and the list of possible constellation symbols in each dimension is recalculated using this new radius to check the possibility of eliminating some of them [19]. The algorithm runs until no unvisited points remain inside the sphere; note that the sphere shrinks with each new point.

We improve the scheme proposed by Hochwald in two ways. Firstly, an estimate of the sphere radius is not required in our scheme. Indeed, we select the sphere radius as the radius of the Babai Point [6], which is a point in the lattice obtained by performing $K$ single-user hard-decisions. It is not necessarily the closest lattice point to the received point but a good choice to start the search. This avoids the waste of time which occurs when the search radius is too small and the search must be restarted. Secondly, we explore the valid points in the arc using the ideas from the Schnorr-Euchner strategy [20] that have been successfully applied to real SD in Multiple Input Multiple Output (MIMO) systems [19]; that is, the list of possible points in (5) is visited in the following order:

$$
\frac{2^{M_{c}}}{2 \pi}\left\lfloor\hat{\theta}_{i}\right\rceil, \frac{2^{M_{c}}}{2 \pi}\left\lfloor\hat{\theta}_{i}\right\rceil+\frac{2 \pi}{2^{M_{c}}}, \frac{2^{M_{c}}}{2 \pi}\left\lfloor\hat{\theta}_{i}\right\rceil-\frac{2 \pi}{2^{M_{c}}}, \frac{2^{M_{c}}}{2 \pi}\left\lfloor\hat{\theta}_{i}\right\rceil+2 \frac{2 \pi}{2^{M_{c}}}, \ldots
$$

where $\lfloor\cdot 7$ operator returns the nearest integer to its argument. In the general case, if each candidate point consists of a radius and an angle then the candidate points from the different circumferences which satisfy (4) are sorted using their distance to the received point. Note that this order does not depend on the sphere radius and the frst visited point is the Babai point.

\section{Sphere DeCoding OF A MC-CDMA System}

To jointly detect an MC-CDMA system using SD, it must be fi stly modelled as a lattice. Previous schemes in MC-CDMA possibly came up as an evolution of DS-CDMA where noise samples are correlated after despreading, and therefore, noise whitening is a common procedure. According to (2), Brunel def nes $\mathbf{M}=\mathbf{C}^{*} \mathbf{G H C}$ and $\mathbf{y}$ becomes a point of a lattice $\Lambda$ with generator matrix $\mathbf{M}$ :

$$
\mathbf{y}=\mathbf{C}^{*} \mathbf{G H C b}+\mathbf{C}^{*} \mathbf{G n}=\mathbf{M b}+\mathbf{C}^{*} \mathbf{G n}
$$

However, $\mathbf{C}^{*} \mathbf{G n}$ is colored noise. To whiten this noise, he carries out the Cholesky factorization of $\mathbf{M}=\mathbf{W}^{*} \mathbf{W}$. Therefore

$$
\mathbf{y}_{\mathbf{w}}=\mathbf{W}^{*-1} \mathbf{W}^{*} \mathbf{W} \mathbf{b}+\mathbf{n}_{\mathbf{w}}
$$

where the new generator matrix turns into $\mathbf{W}$ and $\mathbf{n}_{\mathbf{w}}$ now represents white noise.

Our proposal uses $\mathbf{r}$ instead of $\mathbf{y}$ for the ML sphere decoding operation; with this strategy, the noise remains AWG and no further processing is needed to whiten it. Thus, the above equations can be simplifie as follows. Recalling (1), the received signal is

$$
\mathbf{r}=\mathbf{H C b}+\mathbf{n}
$$

where we defin a new channel matrix $\mathbf{M}^{\prime}=\mathbf{H C}$, which includes spreading and channel effects; indeed, with this approach, despreading and equalizing operations are avoided. The joint ML detection is then performed minimizing the following metric:

$$
\left\|\mathbf{r}-\mathbf{M}^{\prime} \mathbf{b}\right\|^{2}
$$

over all $\mathbf{b}$ valid points in the new lattice $\Lambda^{\prime}$ with generator matrix $\mathbf{M}^{\prime}$. This scheme still performs optimum detection but with a simpler procedure ${ }^{3}$; a complex sphere decoder is also applied to the new lattice in contrast to real one previously proposed [13]. As noted in [13], ML decoding of MC-CDMA systems removes almost all the MUI, allowing near single user performance.

\section{EXPERIMENTS}

We evaluate the proposed transmission system in two-path highly frequency-selective channels with spectral nulls that cancel some subcarriers. Channel impulse response is modeled as a two-tap delayed line $h(t)=\delta(t)+0.7 \delta(t-T)$, where $T$ is the sampling period; similar results are obtained if more dispersive two-ray channels like $h(t)=\delta(t)+(0.7+$ $0.5 j) \delta(t-4 T)$ are considered. At the transmitter, uncoded user bits are mapped into a PSK constellation and these user symbols are spread with Walsh-Hadamard sequences. The system uses $L=32$ subcarriers, and a cyclic prefi is appended to every OFDM symbol before transmission in order to remove Inter-Symbol Interference. At the receiver side, channel estimation is carried out using Pilot-Symbol Assisted Modulation (PSAM) [21]; a preamble of one OFDMsymbol composed of 16 subcarriers conveying pilot symbols is employed to estimate the approximate fat-fading channel per subcarrier.

Figure 2 compares the performance among EGC, MMSE and SD (EGC0, MMSE0, SD lines) in a full-loaded system $(K=L)$. For EGC and MMSE sub-optimum techniques, we also show results after two Parallel Interference Cancellation (PIC) iterations [22] (EGC2 and MMSE2 lines). The sphere

\footnotetext{
${ }^{3}$ Although the number of matrix operations is almost the same, our proposal is conceptually simpler and complex matrix operations are faster than real counterparts in double dimension. For instance, a lattice modeled by a complex matrix $A_{n \times n}$ can be also modeled by a real matrix $A_{2 n \times 2 n}^{\prime}$; the complexity of real Cholesky factorization of the lattice matrix $A_{2 n \times 2 n}^{\prime}$ is $\frac{1}{3} O\left((2 n)^{3}\right)=\frac{8}{3} O\left(n^{3}\right)$ [15] while the complexity of complex Cholesky factorization of $A_{n \times n}$ is $\frac{4}{3} O\left(n^{3}\right)$; the same happens for the matrix inversion operation [14].
} 


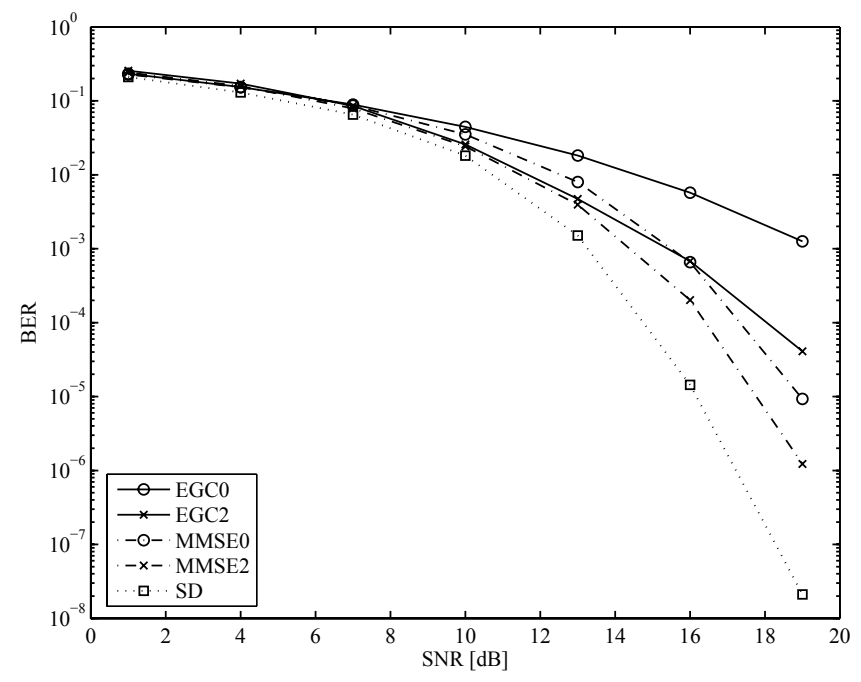

Fig. 2. Comparison between EGC, MMSE and SD in a time-dispersive channel, for a full-loaded system $K=L$. 4-PSK signaling.

decoder remarkably outperforms MMSE for high SNR values, without an estimation of the SNR as required in MMSE technique; we see that the gains from the SD are about two orders of magnitude in Bit-Error-Rate (BER) values. However, for low SNR values, in this case lower than $10 \mathrm{~dB}$, BER performances are similar but decoding complexity of SD is quite a bit higher. Thus, SD algorithms should not be used for many users in very low SNR scenarios because the search time grows with the noise level. In conclusion, this optimum scheme should be used for systems with at least moderate SNR.

In non full-loaded systems $(K<L)$, we can take advantage of MMSE-U equalizing strategy over MMSE at the cost of more complexity. In Figure 3 we plot BER values obtained for different MMSE, MMSE-U and SD methods for $K=32$, 28, 24, 20 users. We can see in this Figure that SD overcomes other methods; for example, we can see that SD for 24 users performs better than MMSE-U for 20 users. Furthermore, the difference between MMSE and MMSE-U in the two PIC iterations case is less when the number of users approaches full-load, so perhaps the additional complexity of MMSE-U is not worthwhile in this case. The expected complexity of $\mathrm{SD}$ algorithm is roughly cubic with the number of users at moderate SNRs; therefore this scheme is particularly suitable for non full-loaded scenarios.

Next, we provide comparisons in terms of computational complexity between real and complex (both original Hochwald's and our proposal) SD. As calculating the covering radius is itself NP-hard, we use the Rogers upper bound of the covering radius for real SD [23]. For the original Hochwald's complex SD we use their radius estimation (which needs an estimate of the noise variance), assuming that it should not be increased with probability 0.8 . Finally, for our proposal on complex SD, we use the radius of the Babai point.

Considering a 16-QAM constellation, the number of real operations performed by the real SD algorithm per visited candidate symbol in $k$-th layer is $(2 k+17)$. On the other hand, the complex SD algorithm performs $(8 k+36)$ real operations [8], [24]. For instance, let us assume an 8-user

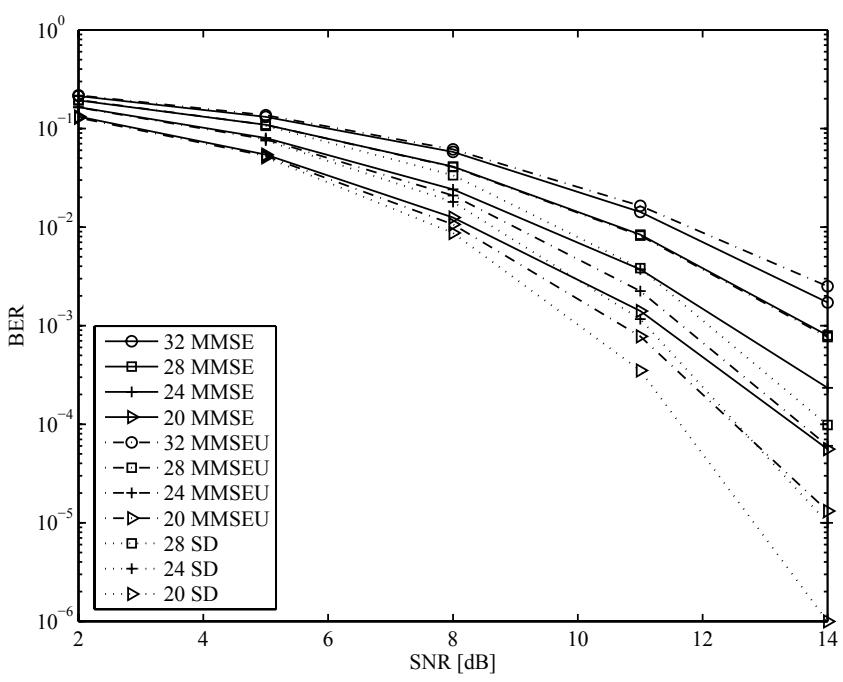

Fig. 3. Comparison between MMSE, MMSE-U and SD for full-loaded and non full-loaded systems in a time-dispersive channel, after two PIC iterations for MMSE and MMSE-U algorithms. The label "32 MMSE" means 32 users and MMSE method, the label "28 MMSE" means 28 users and MMSE method and so on. 4-PSK signaling.

system and that the fir $\mathrm{t}$ lattice point found is the solution. In this case, real SD needs $\sum_{k=1}^{16}(2 k+17)=544$ real operations (note that real SD employs double number of dimensions) while complex SD requires $\sum_{k=1}^{8}(8 k+36)=576$ real operations (5248 and 5376, respectively, for a 32-user system). In other words, complex SD needs on average slightly more real operations than real SD. The complexity of decoding is the sum of two terms: a) the complexity of the lattice set-up, where complex arithmetic is always preferable for complex spreading sequences; b) the complexity of the search, where real SD may be preferable ${ }^{4}$ for rectangular QAM constellations. Therefore, for these constellations, the choice of real or complex SD will be determined by the SNR and the lattice change rate, due to channel or user changes. Further research is needed on this issue.

However, for constellations other than rectangular QAM, real SD wastes time in invalid points since it visits more candidates on average and f nal complexity is much greater, as shown in Figure 4. Also, the use of Schnorr-Euchner enumeration and Babai Point greatly decrease the average number of candidates, which is the difference between Hochwald's and our complex SD. It can be observed that our proposal is especially advantageous in low SNR scenarios. It must be also noted that, in the PSK case with real spreading sequences, the system can not be decoupled in separated real and imaginary parts as in [13] because this may result in an invalid solution.

\section{CONCLUSIONS}

In this paper we develop a new and eff cient complex sphere decoder valid for all constellations and any complex spreading sequence. An upside of this proposal is that it does not require an estimate of the initial search radius. It is also faster than real SD because complex matrix operations need fewer real computations than real counterparts in double dimension. This

\footnotetext{
${ }^{4}$ Here we assume that both complex and real algorithms use the same heuristics to sort the candidate points and to def ne the initial search radius.
} 


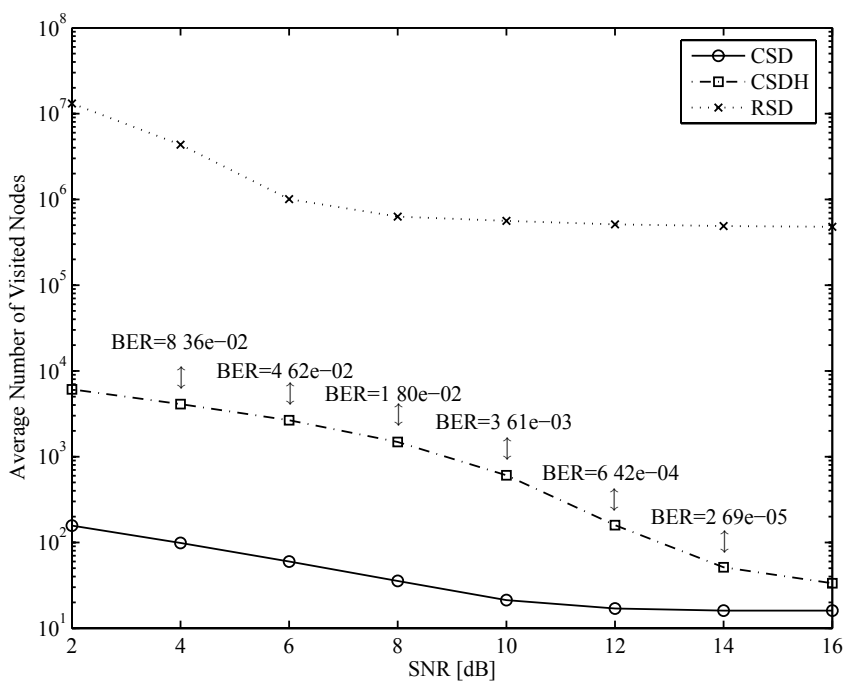

Fig. 4. Comparison among Real SD (RSD), original complex SD (CSDH) and our proposal of complex SD (CSD) in a time-dispersive channel, for an 8-user system and 8-PSK signaling. BER values are specif ed for each SNR point; these values are applicable to the three schemes.

yields lower complexity for the complex lattice set-up, that is, the computation of the lattice matrix and its factorization. Also, it does not waste time on invalid points, as it is the case of the real SD for non-rectangular QAM constellations. In addition, we introduce a new lattice model to decode an MC-CDMA system using a SD algorithm, based on jointly detecting the transmitted symbols directly from the received signal. With this conceptually simpler scheme, the received signal does not need additional processing such as despreading and equalizing, but is directly used to compare with lattice points. Also noise whitening is avoided, since received noise is not transformed. Simulation results have conf rmed the feasibility of the new complex sphere decoder when applied to detect MC-CDMA systems described with our novel lattice. As a future work, it is envisaged that the proposed ideas on lattice models can be extended to pure CDMA systems.

\section{ACKNOWLEDGEMENT}

The authors wish to thank the anonymous reviewers for their detailed and valuable comments that have helped to greatly improve the quality of the paper.

\section{REFERENCES}

[1] K. Fazel and L. Papke, "On the performance of convolutionally-coded CDMA/OFDM for mobile communication system," in Proc. IEEE PIMRC'93, Yokohama, Japan, September 1993, pp. 468-472.

[2] N. Yee, J. P. Linnartz, and G. Fettweis, "Multi-Carrier CDMA in indoor wireless radio networks," in Proc. IEEE PIMRC'93, Yokohama, Japan, September 1993, pp. 109-113.
[3] S. Hara and R. Prasad, "Overview of multicarrier CDMA," IEEE Commun. Mag., vol. 35, no. 12, pp. 126-133, December 1997.

[4] S. Verdú, Multiuser Detection. Cambridge, UK: Cambridge University Press, 1998

[5] S. Kaiser, Multi-Carrier CDMA Mobile Radio Systems. Analysis and Optimization of Detection, Decoding, and Channel Estimation. Munich, Germany: VDI-Verlag, January 1998.

[6] E. Agrell, T. Eriksson, A. Vardy, and K. Zeger, "Closest point search in lattices," IEEE Trans. Inform. Theory, vol. 48, no. 8, pp. 2201-2214, August 2002.

[7] J. Jaldén and B. Ottersten, "On the complexity of sphere decoding in digital communications," IEEE Trans. Signal Processing, vol. 53, no. 4, pp. 1474-1484, April 2005.

[8] B. Hassibi and H. Vikalo, "On the sphere-decoding algorithm I. expected complexity," IEEE Trans. Signal Processing, vol. 53, no. 8, pp. 28062818, August 2005.

[9] M. Pohst, "On the computation of lattice vectors of minimal length, successive minima and reduced bases with applications," ACM SIGSAM Bulletin, vol. 15, no. 1, pp. 37-44, February 1981.

[10] E. Viterbo and E. Biglieri, "A universal decoding algorithm for lattice codes," in Proceedings GRETSI, Juan-les-Pins, France, September 1993, pp. 611-614.

[11] E. Viterbo and J. J. Boutros, "A universal lattice code decoder for fading channels," IEEE Trans. Inform. Theory, vol. 45, no. 5, pp. 1639-1642, 1999.

[12] B. M. Hochwald and S. ten Brink, "Achieving near-capacity on a multiple-antenna channel," IEEE Trans. Commun., vol. 51, no. 3, pp. 389-399, March 2003.

[13] L. Brunel, "Multiuser detection techniques using maximum likelihood sphere decoding in multicarrier CDMA systems," IEEE Trans. Wireless Commun., vol. 3, no. 3, pp. 949-957, May 2004.

[14] L. W. Ehrlich, "Complex matrix inversion versus real," Commun. ACM, vol. 13 , no. 9, pp. 561-562, 1970.

[15] G. H. Golub and C. F. V. Loan, Matrix Computations, 3rd ed. Baltimore, MD, USA: Johns Hopkins University Press, 1996.

[16] S. Kaiser, "On the performance of different detection techniques for OFDM-CDMA in fading channels," in IEEE Global Telecommunications Conference, vol. 3, 1995, pp. 2059-2063.

[17] D. N. Kalofonos, M. Stojanovic, and J. G. Proakis, "On the performance of adaptive MMSE detectors for a MC-CDMA system in fast fading rayleigh channels," in Proc. of Personal, Indoor, and Mobile Radio Conference (PIMRC), Boston, MA, USA, 1998, pp. 1309-1313.

[18] J. F. Hélard, J. Y. Baudais, and J. Citerne, "Linear MMSE detection technique for MC-CDMA," Electronics Letters, vol. 36, no. 7, pp. 665666, March 2000.

[19] A. M. Chan and I. Lee, "A new reduced-complexity sphere decoder for multiple antenna systems," in IEEE International Conference on Communications, 2002. ICC 2002, vol. 1, 28 April-2 May 2002, pp. 460-464.

[20] C. P. Schnorr and M. Euchner, "Lattice basis reduction: Improved practical algorithms and solving subset sum problems." Mathematical Programming, vol. 66, pp. 181-191, July 1997.

[21] J. K. Cavers, "An analysis of pilot symbol assisted modulation for rayleigh fading channels," IEEE Trans. Veh. Technol., vol. 40, no. 4, pp. 686-693, November 1991.

[22] P. Patel and J. Holtzman, "Analysis of simple successive interference cancellation scheme in DS/CDMA system," IEEE J. Select. Areas Commun., vol. 12, June 1994.

[23] J. H. Conway and N. J. A. Sloane, Sphere Packings, Lattices and Groups, 3rd ed. Springer Verlag, 1999.

[24] H. Vikalo and B. Hassibi, "On the sphere-decoding algorithm II. generalizations, second-order statistics, and applications to communications," IEEE Trans. Signal Processing, vol. 53, no. 8, pp. 2819-2834, August 2005. 